\title{
Una nueva especie de Puya (Bromeliaceae) de los páramos cercanos a Bogotá, Colombia
}

\author{
Santiago Madriñán
}

Laboratorio de Botánica y Sistemática, Departamento de Ciencias Biológicas, Universidad de los Andes, Bogotá, Colombia

\begin{abstract}
Resumen
Se describe una nueva especie de Puya de los páramos cercanos a Bogotá, Colombia. Puya loca difiere de todas las especies conocidas del género Puya por el envés de sus brácteas y sépalos ferrugíneo-tomentosos y pétalos de color púrpura intenso. Esta nueva especie forma masas densas de rosetas en acantilados rocosos. Hasta el momento es endémica de pequeños parches aislados de páramos al noreste de Bogotá, en el complejo de páramos de Chingaza. La nueva especie se compara con otras que tienen flores de color similar y crecen en los alrededores de Bogotá y en otras áreas.
\end{abstract}

Palabras clave: Bromeliaceae, complejo Chingaza, cerro Tunjaque, páramos, Puya loca.

\section{A new species of Puya (Bromeliaceae) from the páramos near Bogotá, Colombia}

\begin{abstract}
A new species of Puya from the páramos in the vicinity of Bogotá, Colombia, is described. Puya loca differs from all known species of the genus Puya by its ferrugineo-tomentose bracts and sepals abaxially, and deep-purple colored petals. It is, until now, endemic to small isolated patches of páramos North-East of Bogotá, in the Chingaza Páramo Complex. It forms dense mats of rosettes on rocky cliffs. The new species is compared to other known species from the surroundings of Bogotá, as well as to species with similar colored flowers from other regions.
\end{abstract}

Key words: Bromeliaceae, Chingaza Complex, Cerro Tunjaque, Páramos, Puya loca.

\section{Introducción}

El género Puya Molina (Bromeliaceae) comprende especies de plantas terrestres arrosetadas acaules (raramente caulescentes), con inflorescencias terminales, monocaules y hapaxánticas, o ramificadas basitónicamente que forman simpódios pluricaules pleonánticos, y flores vistosas (Smith \& Downs, 1974). Sus hojas provistas de espinas a lo largo de las márgenes sugieren el nombre común de "puya" que estas plantas reciben en Hispanoamérica y que fue tomado por el abate chileno Juan Ignacio Molina (1740-1829) para describir Puya chilensis Molina (Molina, 1782).

En su revisión más reciente del género, Lyman B. Smith y Robert J. Downs (1974) describen 168 especies y seis variedades. Desde esta revisión, entre los años 1976 y 2013, dos especies preexistentes se han considerado híbridos naturales y 52 especies y cuatro taxones infraespecíficos se han descrito como nuevos, para un total de 222 especies (incluidos tres híbridos naturales) y diez taxones infraespecíficos aceptados (sensu Butcher \& Gouda, 2015). En la Encyclopedia of Bromeliads y demás páginas de internet administradas por Eric J. Gouda (2015), se sugieren varias especies nuevas, lo que ratifica la acusada tendencia al aumento de su número (Figura 1). Lyman
B. Smith y Robert J. Downs (1974) subdividieron el género en dos subgéneros de acuerdo a la composición de sus inflorescencias: el subgénero Puya, con las ramas de sus inflorescencias estériles en su parte apical (solo nueve especies), y Puyopsis, con las ramas fértiles a todo lo largo del eje.

La mayoría de las especies del género son endémicas de la cordillera de los Andes, con unas pocas especies presentes en las montañas del Escudo Guayanés, y solo dos especies presentes en Costa Rica, la endémica Puya dasylirioides Standl., y Puya floccosa (Linden) E.Morren ex Mez de amplia distribución compartida entre los Andes de Colombia (La Macarena), Venezuela, y la Cordillera de la Costa y el Escudo Guayanés venezolano (Smith \& Downs, 1974). Los países con mayor número de especies reportadas son Perú y Bolivia, con 75 y 52, respectivamente. En Colombia se han registrado 37 especies, de las cuales 26, así como tres variedades, son endémicas (Betancur \& Callejas, 1997; Betancur, 2015; Gouda, et al., 2015; Tabla 1).

\footnotetext{
Correspondencia:

Santiago Madriñán, samadrin@uniandes.edu.co

Recibido: 26 de abril de 2015

Aceptado: 23 de junio de 2015
} 


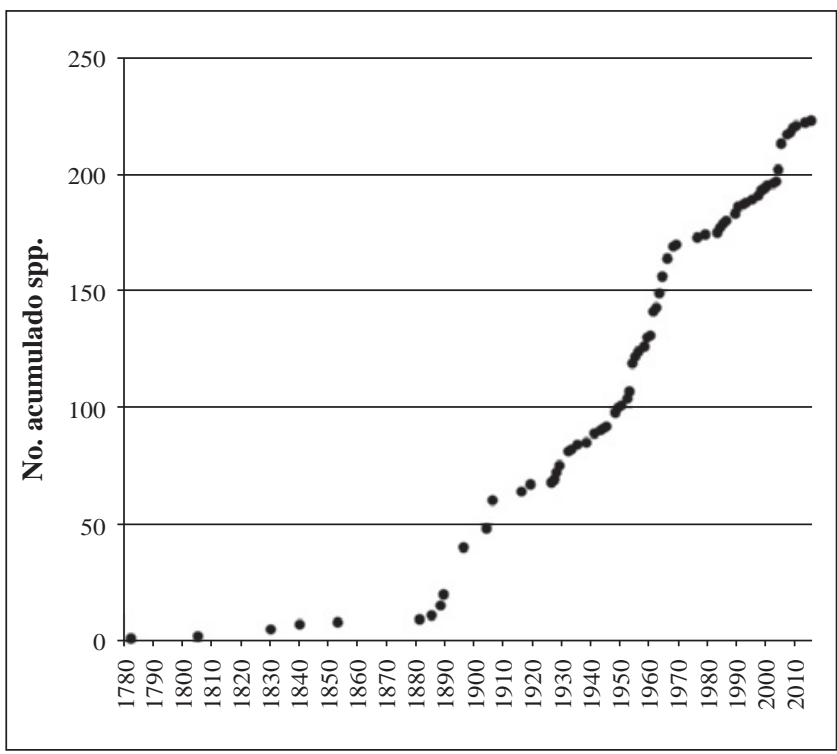

Figura 1. Acumulación de especies aceptadas de Puya desde que fue nombrada la primera especie en 1782 hasta 2015.
En estudios moleculares recientes de esta familia llevados a cabo por Givnish, et al. (2007), y los adelantados por Jabaily \& Sytsma (2010, 2012) en la especie Puya, los autores sustentan parcialmente la subdivisión genérica pro-puesta por Smith \& Downs (1974); en ellos se reconocen dos "grupos" (grados) parafiléticos: el primero, de divergencia temprana, incluye la mayoría de las especies del subgénero Puya; en el segundo se puede evidenciar un grado para-filético "basal” y un grupo monofilético en el cual se encuentran las especies tropicales de alta montaña de los Andes del Norte.

La mayoría de las especies de Puya confinadas a los Andes del Norte se encuentra en los páramos y constituye un grupo monofilético. El autor y los colaboradores dataron el origen del género Puya a 9,10 Ma y el clado norandino en particular, a 0,26-0,80-1,58 Ma; dado el número de especies pertenecientes a este clado (46), se estableció una tasa de diversificación extremadamente alta $(\mathrm{r}=12,06-3,92-1,98)$, muy superior a cualquier tasa de diversificación conocida (Madriñán, et al., 2013).

Tabla 1. Especies de Puya presentes en Colombia y datos de su distribución. Adaptado de Gouda et al. (2015) y Betancur (2015). El color de los pétalos proviene de diversas fuentes y es aproximado.

\begin{tabular}{|c|c|c|c|c|c|}
\hline Especie & $\begin{array}{l}\text { Elevación } \\
\text { (m.s.n.m) }\end{array}$ & $\begin{array}{l}\text { Endémica en } \\
\text { Colombia }\end{array}$ & Departamentos & Otros países & $\begin{array}{l}\text { Color de } \\
\text { pétalos }\end{array}$ \\
\hline Puya alpicola L.B.Sm. & $3.140-3.900$ & & $\begin{array}{l}\text { Magdalena (Sierra Nevada de Santa } \\
\text { Marta) }\end{array}$ & & \\
\hline P. antioquensis L.B.Sm. \& Read & $2.980-3.680$ & $\mathrm{X}$ & Antioquia & & \\
\hline P. aristeguietae L.B. Sm.* & $2.700-3.550$ & & Arauca, Arauca & Venezuela & Verde pálido \\
\hline P. barkleyana L.B.Sm. & $2.800-3.693$ & $\mathrm{X}$ & Boyacá, Santander & & \\
\hline P. bicolor Mez & $2.423-3.500$ & $\mathrm{X}$ & $\begin{array}{l}\text { Boyacá, Cundinamarca, Norte de } \\
\text { Santander }\end{array}$ & & Verde lila, azul \\
\hline P. boyacana Cuatrec. & $3.300-3.400$ & $\mathrm{X}$ & Boyacá & & Verde azul \\
\hline P. brachystachya (Baker) Mez & 2.880 & $\mathrm{X}$ & $\begin{array}{l}\text { Magdalena (Sierra Nevada de Santa } \\
\text { Marta) }\end{array}$ & & Crema, verde pálido \\
\hline P. clava-herculis Mez \& Sodiro & $3.200-3.800$ & & Cundinamarca, Nariño & Ecuador & Azul verde \\
\hline P. cleefii L.B.Sm. \& Read & 3.700 & $\mathrm{X}$ & Boyacá & & Verde pálido \\
\hline P. cryptantha Cuatrec. & $3.100-3.950$ & $\mathrm{X}$ & $\begin{array}{l}\text { Antioquia, Boyacá, Cundinamarca, } \\
\text { Nariño, Norte de Santander }\end{array}$ & & Verde, azul pálido \\
\hline P. cuatrecasasii L.B.Sm. & $2.800-4.300$ & $\mathrm{X}$ & Cauca, Nariño, Tolima, Valle & & Blanco cremoso \\
\hline P. dichroa L.B.Sm. \& Read & 3.750 & $\mathrm{X}$ & Boyacá & & Azul oscuro \\
\hline P. exuta L.B.Sm. \& Read & $1.990-2.970$ & $\mathrm{X}$ & Boyacá, Santander & & \\
\hline $\begin{array}{l}\text { P. floccosa (Linden) E.Morren } \\
\text { ex Mez }\end{array}$ & $211-2.500$ & & $\begin{array}{l}\text { Boyacá, Caquetá, Cauca, } \\
\text { Cundinamarca, Guaviare, Huila, Meta, } \\
\text { Norte de Santander, Santander, Tolima }\end{array}$ & $\begin{array}{c}\text { Costa Rica } \\
\text { Venezuela Brasil }\end{array}$ & $\begin{array}{l}\text { Azul con verde, } \\
\text { estambres azules }\end{array}$ \\
\hline P. furfuracea (Willd.) L.B.Sm. & $2.390-3.200$ & $\mathrm{X}$ & Cauca, Nariño & & \\
\hline P. gargantae L.B.Sm. & $3.100-3.700$ & $\mathrm{X}$ & Norte de Santander & & \\
\hline$P$. gigas André & $3.000-3.300$ & $\mathrm{X}$ & Nariño & & \\
\hline P. goudotiana Mez & $2.760-3.550$ & $\mathrm{X}$ & $\begin{array}{l}\text { Boyacá, Cundinamarca, Norte de } \\
\text { Santander, Santander }\end{array}$ & & Verde azuloso \\
\hline
\end{tabular}




\begin{tabular}{|c|c|c|c|c|c|}
\hline Especie & $\begin{array}{l}\text { Elevación } \\
(\mathrm{m} . \text { s.n.m) }\end{array}$ & $\begin{array}{l}\text { Endémica en } \\
\text { Colombia }\end{array}$ & Departamentos & Otros países & $\begin{array}{l}\text { Color de } \\
\text { pétalos }\end{array}$ \\
\hline P. grantii L.B.Sm. & $2.531-3.650$ & $\mathrm{X}$ & Boyacá, Cesar & & \\
\hline P. grubbii L.B.Sm. & $2.050-2.200$ & $\mathrm{X}$ & Boyacá & & Verde grisáceo \\
\hline P. hamata L.B.Sm. & $2.500-3.750$ & & Cauca, Nariño, Putumayo, Tolima, Valle & Ecuador, Perú & Azul oscuro \\
\hline P. horrida L.B.Sm. \& Read & 3.450 & $\mathrm{X}$ & Cundinamarca & & \\
\hline P. killipii Cuatrec. & $3.000-3.600$ & & Norte de Santander, Santander & Venezuela & Azul \\
\hline P. lehmanniana L.B.Sm. & $1.800-3.200$ & & Cauca, Nariño & Ecuador & Azul oliváceo \\
\hline P. lineata Mez & $2.700-3.700$ & $\mathrm{X}$ & Cundinamarca & & Azul \\
\hline P. loca Madriñán sp. nov. & $3.200-3.550$ & $\mathbf{X}$ & Cundinamarca & & Púrpura intenso \\
\hline P. nitida Mez & $2.700-3.700$ & $\mathrm{X}$ & Boyacá, Cundinamarca, Santander & & Verde claro con lila \\
\hline $\begin{array}{l}\text { P. nitida var. glabrior L.B.Sm. } \\
\text { \& Read }\end{array}$ & 3.300 & $\mathrm{X}$ & Boyacá & & \\
\hline P. nivalis Baker & 3.250 & $\mathrm{X}$ & $\begin{array}{l}\text { Magdalena (Sierra Nevada de Santa } \\
\text { Marta) }\end{array}$ & & Crema, verde pálido \\
\hline P. occidentalis L.B.Sm. & $3.300-3.700$ & $\mathrm{X}$ & Valle & & \\
\hline $\begin{array}{l}\text { P. ochroleuca Betancur \& } \\
\text { Callejas }\end{array}$ & $2.850-3.900$ & $\mathrm{X}$ & Antioquia, Caldas, Quindío, Tolima & & Amarillo pálido \\
\hline P. roldanii Betancur \& Callejas & $2.700-3.240$ & $\mathrm{X}$ & Antioquia & & Verde azul \\
\hline P. sanctae-martae L.B.Sm. & $2.600-3.200$ & $\mathrm{X}$ & $\begin{array}{l}\text { Magdalena, La Guajira (Sierra Nevada } \\
\text { de Santa Marta y Serranía del Perijá) }\end{array}$ & & \\
\hline P. santanderensis Cuatrec. & $2.850-3.500$ & $\mathrm{X}$ & Norte de Santander & & $\begin{array}{l}\text { Blanco con tinte verde } \\
\text { amarillento }\end{array}$ \\
\hline P. santosii Cuatrec. & $2.800-4.285$ & $\mathrm{X}$ & Arauca, Boyacá, Cundinamarca, Meta & & Azul verdoso \\
\hline $\begin{array}{l}\text { P. santosii var. verdensis } \\
\text { Cuatrec. }\end{array}$ & $3.100-3.700$ & $\mathrm{X}$ & Cundinamarca & & \\
\hline P. thomasiana André & $2.450-2.590$ & & Nariño & Ecuador & Azul verdoso \\
\hline P. trianae Baker & $3.000-4.100$ & & $\begin{array}{l}\text { Antioquia, Arauca, Boyacá, } \\
\text { Cundinamarca, Quindío, Santander, } \\
\text { Tolima, Valle }\end{array}$ & Venezuela & Crema, verde pálido \\
\hline $\begin{array}{l}\text { P. trianae var. amplior } \\
\text { L.B.Sm.\& Read }\end{array}$ & 4.000 & $\mathrm{X}$ & Boyacá & & \\
\hline P. venezuelana L.B.Sm. & $3.255-3.960$ & & Arauca, Cesar, Norte de Santander & Venezuela & Amarillo verdoso \\
\hline P. vestita André & $3.200-3.400$ & & Nariño, Putumayo & Ecuador & Verde pálido \\
\hline
\end{tabular}

*Nota: Espécimen recolectado por A.M. Cleef (10026) en 1973 en el Parque Nacional Natural El Cocuy, determinado por L. B. Smith, 1975.

Los estudios moleculares de las especies chilenas hechos mediante la técnica de polimorfismos en la longitud de los fragmentos amplificados (Amplified Fragment Length Polymorphism, AFLP) (Jabaily \& Sytsma, 2010; Schulte, et al., 2010), agrupan a algunas de las especies estudiadas según el color de sus pétalos, estableciendo un clado de Puya con flores amarillas y otro con flores azules y evidenciando eventos de hibridación e introgresión entre ellos. Quizás uno de los caracteres más sobresalientes en la identificación de especies de Puya es el color de los pétalos, los cuales se presentan en fuertes tonos de blanco, amarillo, verde, azul o lila a púrpura y en tonos intermedios. Comúnmente, el color de los pétalos contrasta con el color de los sépalos. Estos colores y contrastes sugieren un síndrome de polinización ornitofílico, en tanto que la disposición de las flores en inflorescencias sobresalientes, con las flores expuestas sin estructuras de percha y presencia de néctar copioso, son típicas de una polinización mediada por colibríes, como se ha observado frecuentemente. En un estudio reciente sobre polinizadores de especies de Puya en Chile se estableció una correlación entre la ausencia de flores apicales en las ramas secundarias (subgénero Puyopsis), los pequeños volúmenes con alta concentración de néctar y la polinización por colibríes (Hornung-Leoni, et al., 2013). Por otro lado, dicho estudio relacionó especies de Puya, subgénero Puya, con los ápices estériles de las ramas de la inflorescencia que forman una percha, y la baja concentración y altos volúmenes de néctar con la polinización por Passerina generalistas.

Las inflorescencias inmaduras y los primordios foliares de las especies de Puya son el alimento predilecto del oso de anteojos (Tremarctos ornatus) en la puna (RivadeneiraCanedo, 2008) y los páramos (DeMay, et al., 2014). En este 
último estudio se sugiere que la topografía puede ser un factor que influye en la intensidad del forrajeo por parte de los osos, por lo que aquellas plantas localizadas en pendientes estarían sujetas a un menor grado de herbivoría. Es muy probable que la presencia de espinas en las hojas de las especies de Puya constituya una defensa de las inflorescencias inmaduras ante el forrajeo de los osos de anteojos y otros herbívoros y omnívoros andinos. David J. Mabberley (1987) menciona que en Chile las puyas son erradicadas, pues pueden atrapar ovejas o aves en sus rosetas (por efecto de sus espinas orientadas hacia el interior de la roseta), lo cual puede constituir una fuente y una forma de absorber nutrientes por vía foliar.

\section{Materiales y métodos}

En el marco del proyecto de determinación de la interfaz entre bosque y páramo de los complejos de páramos de Colombia liderado por el Instituto Alexander von Humboldt, durante los meses de octubre a diciembre del 2014 un grupo de trabajo encabezado por el autor estudió la composición de la edafofauna, la herpetofauna, la ornitofauna y la vegetación a lo largo de cinco transectos en el complejo de páramos de Chingaza en cercanías de Bogotá. Uno de estos transectos se ubicó en el cerro Tunjaque (04 $38^{\circ}$ 21,49” N, 73 55’53,76” O), suroriente del municipio de La Calera, Cundinamarca, a $13 \mathrm{~km}$ de distancia horizontal hacia el oriente de la ciudad de Bogotá. Este cerro es un macizo aislado de otras masas montañosas de altura por valles profundos y laderas escarpadas, y forma una isla de páramo de aproximadamente $2 \mathrm{~km}^{2}$ ubicada a una distancia horizontal de aproximadamente $10 \mathrm{~km}$ en los cuatro puntos cardinales con relación a los páramos más cercanos. Su altura máxima es de 3.550 m.s.n.m. (Figuras 2 y 3). El cerro Tunjaque se encuentra protegido por estar en la reserva privada Tierraleja, propiedad del Sr. Antonio Piñeros.

En la parte más alta del cerro, en un acantilado rocoso, se encontró una población de una especie de Puya, la cual se considera aquí como una especie nueva por sus rosetas cespitosas pequeñas, sus flores grandes, el color de sus brácteas y sépalos ferrugíneo-tomontosos y en particular por sus pétalos de color púrpura intenso. A unos $300 \mathrm{~m}$ cuesta abajo, en las paredes rocosas de una cascada, se encontró una segunda población aislada. Durante las exploraciones en el páramo aledaño de Buenos Aires (Cerro Verde, 04 43’ 42,22” N, 73 53’ 23,54” O, 3.500 m.s.n.m.) por la vía hacia Piedras Gordas en el Parque Nacional Natural Chingaza, se encontró otra población aislada con unos pocos individuos y la presencia de algunos otros de Puya nitida Mez con los pétalos de color rosado, los cuales sugieren un posible híbrido natural entre esta última y la nueva especie aquí descrita.

Se realizaron experimentos de germinación de semillas de la especie nueva y su híbrido putativo mediante el aislamiento de semillas, esterilización en solución de hipoclorito al 10 \% durante 10 minutos y siembra en platos de Petri con algodón humedecido.

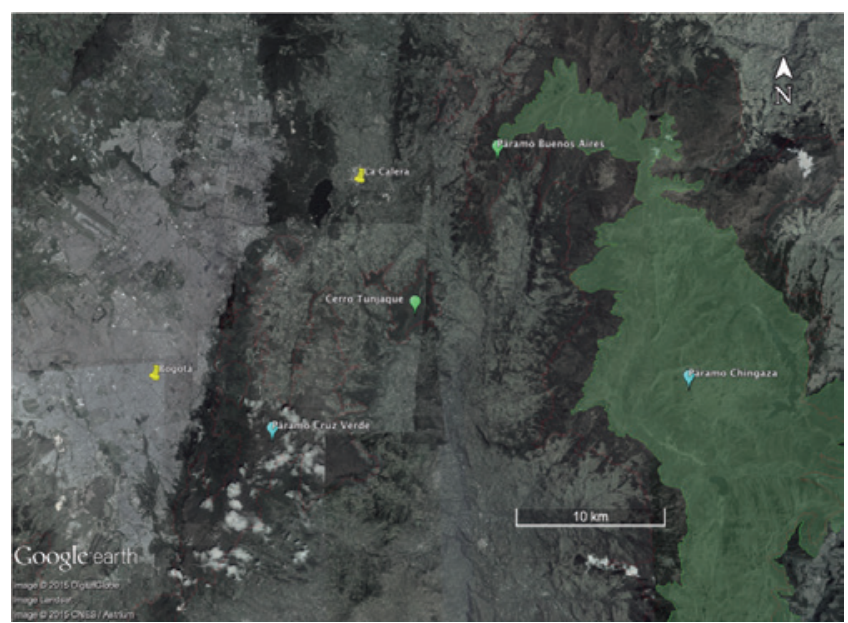

Figura 2. Imagen satelital del área de distribución de la especie Puya loca. Los marcadores verdes indican las localidades donde se han encontrado poblaciones de la especie y los amarillos, páramos grandes circundantes. El área resaltada en verde corresponde al Parque Natural Nacional Chingaza. Imagen de GoogleEarth.

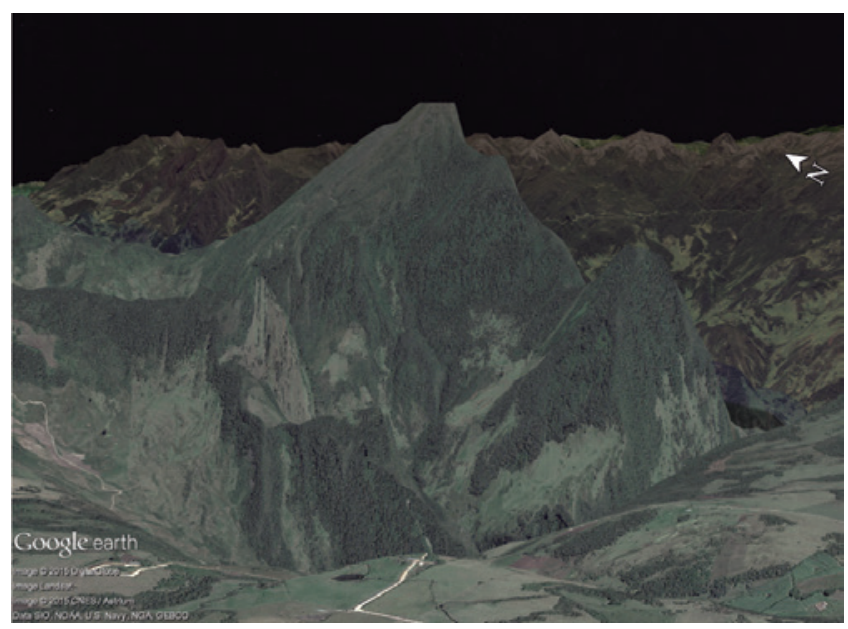

Figura 3. Vista digital sobrepuesta en una imagen satelital del cerro Tunjaque desde el páramo de Cruz Verde hacia el nororiente, en la cual se evidencia su carácter aislado, rodeado de valles profundos. Imagen de GoogleEarth.

La descripción de la especie se basa en el espécimen tipo y en las observaciones en campo de especímenes vivos de la población tipo. Los caracteres y su orden en la descripción siguen las sugerencias de Eric J. Gouda para la familia Bromeliaceae (1998; Scharf \& Gouda, 2008).

\section{Taxonomía}

Puya loca Madriñán sp. nov. (Figuras 4 y 5)

Tipo: COLOMBIA. Cundinamarca: Municipio La Calera, cerro Tunjaque, reserva Tierraleja, $04^{\circ} 38^{\prime} 21,49^{\prime}$ N, $73^{\circ} 55^{\prime}$ 53,76” O, 3.550 m, 30 noviembre de 2014, S. Madriñán \& A. Piñeros 2910 (holotipo: ANDES; isotipo: COL). 

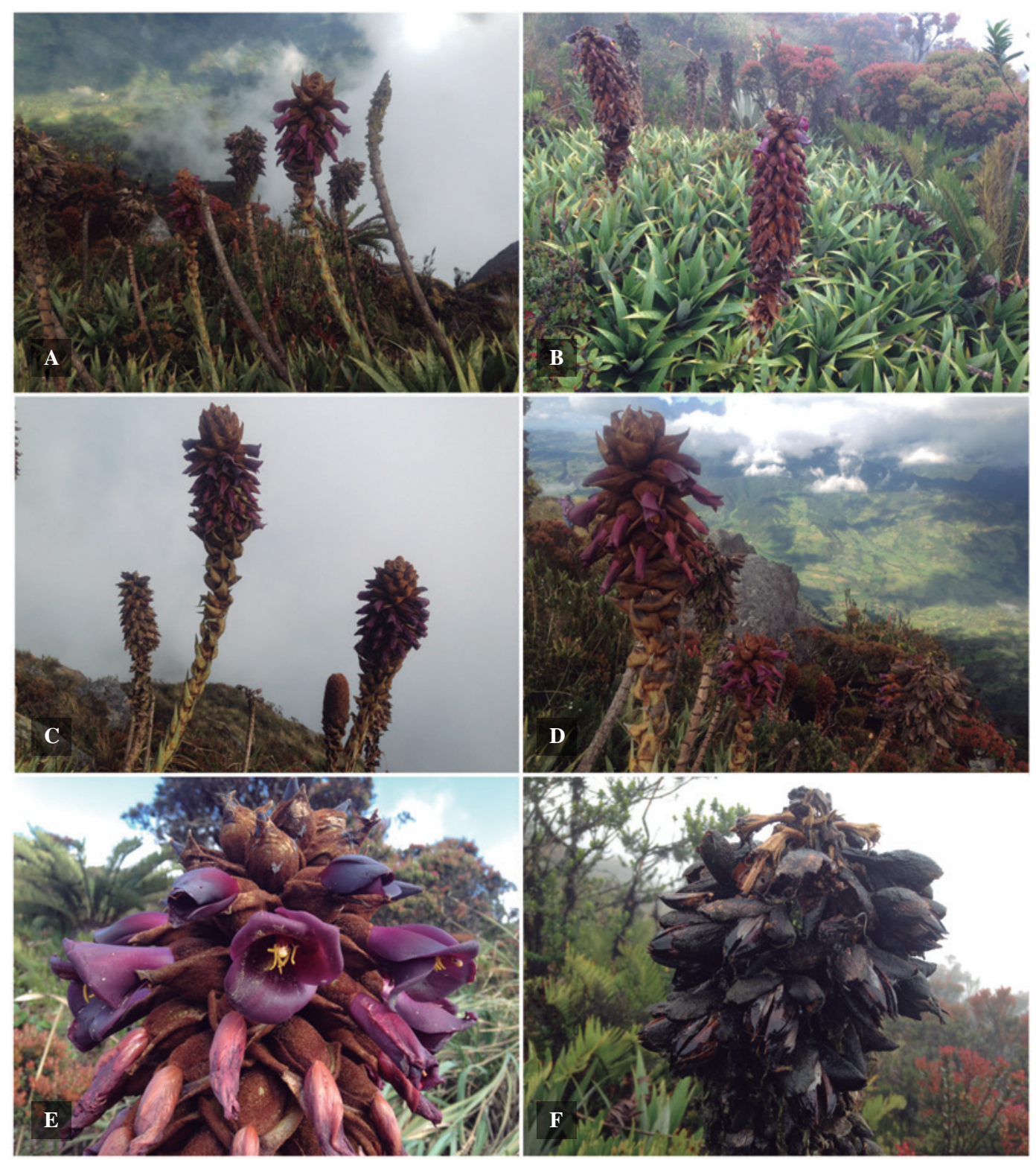

Figura 4. Fotografías de Puya loca en su localidad típica, el cerro Tunjaque. A-D. hábitta y hábito. E. inflorescencia. F. infrutescencia. Fotografías de S. Madriñán.

Diagnosis: Puya loca difiere de todas las demás especies conocidas de Puya por sus rosetas cespitosas pequeñas que forman masas densas de hasta $3 \mathrm{~m}$ de diámetro, inflorescencias tres veces más largas que la roseta, brácteas y sépalos ferrugíneo-tomentosos y flores de pétalos grandes de color púrpura intenso.

Planta acaule, simpodial, cespitosa, que forma conjuntos densos de rosetas, de 25 a $40 \mathrm{~cm}$ de alto, densas, polísticas con cerca de 30 hojas maduras, verde lustrosas, con inflorescencia tres veces más larga que la roseta. Hojas coriáceas, de cerca de $30 \mathrm{~cm}$ de largo, mucho más cortas que las inflorescencias; vainas carnosas, glabras, discoloras con la lámina, blancas, deltoides, no pecioladas, no infladas, de 3 a $4 \mathrm{~cm}$ de largo, 4 a $5 \mathrm{~cm}$ de ancho, de margen entera, membranácea; láminas homomórficas, arqueadas, rígidas, coriáceas, acanaladas, lanceoladas, de 25 a $30 \mathrm{~cm}$ de largo, $4 \mathrm{a} 5 \mathrm{~cm}$ de ancho en la base, aserradas, espinas distanciadas 10 a $15 \mathrm{~mm}$, hialinas incoloras, antrorsas, de 2,4 a 3,0 mm de largo, delgadas; láminas atenuadas hacia el ápice, ápice agudo, discoloras, verde brillantes y glabras por el haz, verde opacas y densamente lepidotas por el envés, con escamas densas, minúsculas, grisáceas. Inflorescencia terminal, simple, espigada, densa, porción fértil de 25 a 30 $\mathrm{cm}$ de largo, 15 a $18 \mathrm{~cm}$ de ancho, subcilíndrica, longitud total de la inflorescencia de 70 a $85 \mathrm{~cm}$, ferrugíneotomentosa; pedúnculos elongados, erectos a levemente curvos, de 45 a $55 \mathrm{~cm}$ de largo, 3 a $4 \mathrm{~cm}$ de diámetro, 

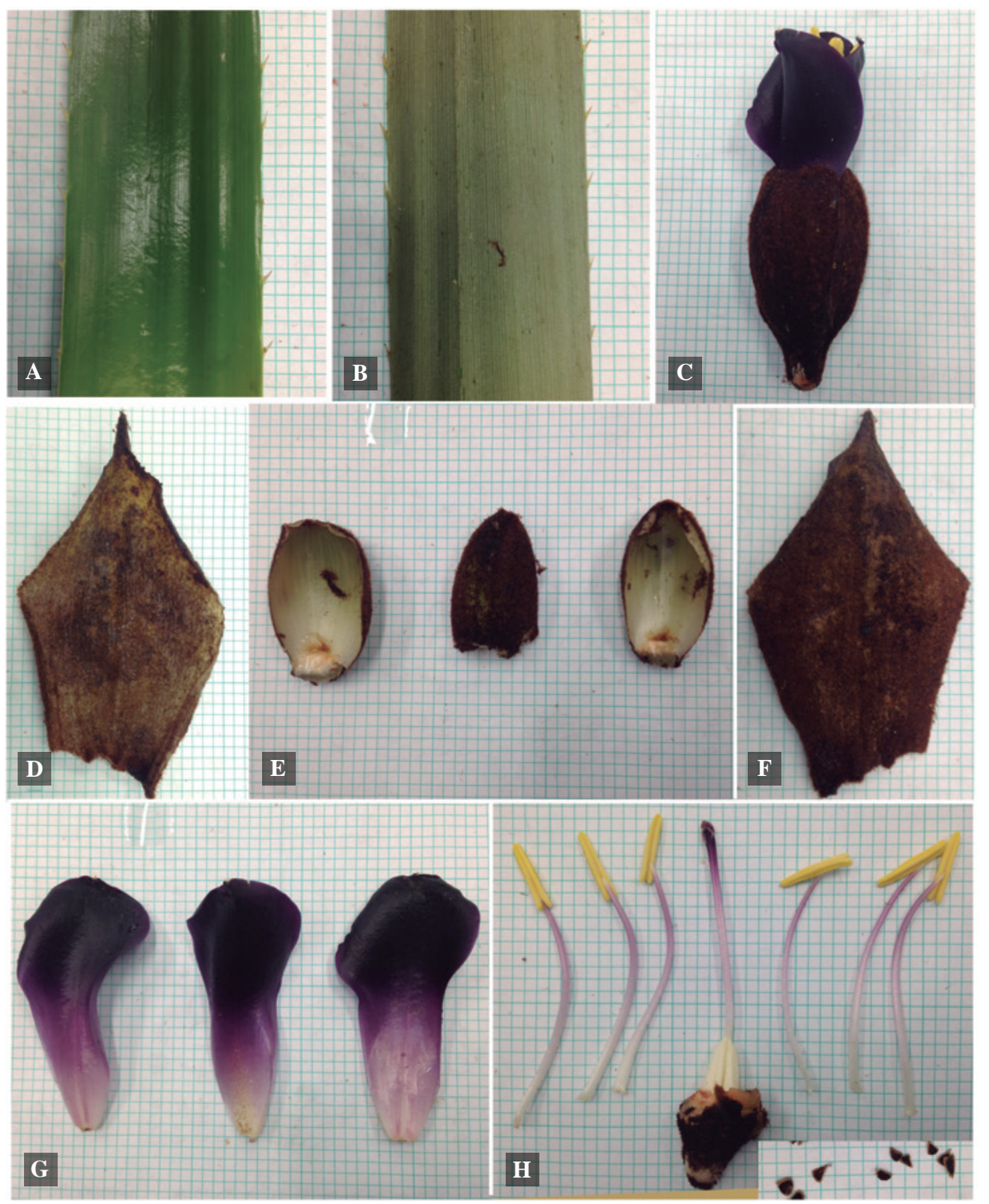

Figura 5. Detalles de las hojas y disección floral de Puya loca. A. Porción medial de una hoja (haz). B. Porción medial de una hoja (envés). C. Flor entera. D. Bráctea floral (haz). E. Sépalos. F. Bráctea floral (envés). G. Pétalos. H. Estambres y receptáculo con pistilo (inserto semillas). Escalas: $10 \mathrm{~cm}$. Fotografías de S. Madriñán.

densamente ferrugíneo-tomentosos; brácteas pedunculares foliáceas en la base, imbricadas a laxamente imbricadas acrotónicamente, coriáceas, lanceoladas, finamente aserradas, ápice agudo, cortas en relación con la longitud del pedúnculo, diminutamente lepidotas, verde grisáceas; eje parcialmente expuesto, rígido, recto a levemente flexuoso, terete, densamente ferrugíneo-tomentoso, glabrescente, epidermis verde clara; espigas erectas, polísticas, densas, con 20 a 50 flores, solo unas 6 a 9 en antesis, linear-lanceoladas, subcilíndricas, ápice redondeado; raquis oculto; brácteas florales abundantes, amplexifloras las flores, imbricadas, membranáceas, sin nerviación prominente, sin carina, ampliamente obovadas a romboides, margen entero, ápice acuminado, incurvadas, de 7 a $8 \mathrm{~cm}$ de largo, 4,5 a $5 \mathrm{~cm}$ de ancho, varias veces más largas que los entrenudos, un poco más largas que los sépalos, márgenes delgadas, densamente ferrugíneo-tomentosas por ambas caras, indumento persistente, epidermis verde. Flores péndulas, de 7,5 a 8,5 cm de largo, subsésiles; receptáculo obcónico de 1,5 a 2,0 cm de largo, 1,5 a $1,8 \mathrm{~cm}$ de ancho, densamente ferrugíneo-tomentoso; sépalos rígidos, carnosos, sin nerviación prominente, ovado oblongos a orbiculares, simétricos, margen entera, desarmados, ápice obtuso, fuertemente incurvados, iguales, de 3,6 a 3,8 cm de largo, 6,1 a 6,3 cm de ancho, margen gruesa, sin carina, no fusionados, glabros por el haz, densamente ferrugíneotomentosos por el envés, epidermis crema-verdosa; pétalos, levemente carnosos, espatulados, en la base envolviendo los estambres, el ápice de la lámina amplio, redondeado, 
reflexos, de 6,1 a 6,3 $\mathrm{cm}$ de largo, 1,5 a $1,7 \mathrm{~cm}$ de ancho en su porción medial, de 3,0 a 3,3 cm de ancho en su porción más ancha, libres, color lila pálido en la base, intensificándose acrotónicamente a un color púrpura intenso en la porción apical, sin lígulas; estambres igual de largos al pistilo, un poco más cortos que los pétalos; filamentos aplanados en la base, teretes hacia el ápice, no plicados, iguales, curvos, de 5,1 a 5,2 cm de largo, libres, blancos en la base, cambiando a lila acrotónicamente, anteras dorsifijas, unidas al filamento a un tercio de su longitud, versátiles, tubulares a levemente sagitadas, de 1,2 a 1,5 cm de largo; pistilo igual de largo a los estambres, ovario súpero, ovoide a piramidal, estriado, de 1,0 a 1,2 cm de largo, 0,8 cm de ancho en la base, contraído hacia el estilo, estilo, delgado, elongado, varias veces más largo que el ovario, blanco en la base, cambiando a lila acrotónicamente y terminando en un estigma púrpura intenso, estigmas lineares, espiralados. Fruto con pedicelo elongado post-antesis, de 2,0 a 2,5 cm de largo, $0,5 \mathrm{~cm}$ de diámetro, cápsula globosa, de 2,5 a 3,0 cm de largo, 2,0 a 2,3 cm de diámetro, apocárpica secundaria, dehiscencia septicida, levemente excediendo las brácteas florales, ápice atenuado, glabro, marrón a negro lustroso. Semillas cuneiformes, de 3,0 mm de largo, 2,0 mm de ancho, rojizas, aladas, alas marrón claro.

Distribución y hábitat: esta especie se ha encontrado solamente en los páramos aislados del cerro Tunjaque y Cerro Verde en el municipio de La Calera aledaño a la ciudad de Bogotá, entre los 3.500 y los 3.550 m.s.n.m. Una población azonal se encontró a 2.250 m.s.n.m. Es muy probable que se encuentre, igualmente, en el cerro Los Quemados (04 $42^{\prime} 2,71^{\prime} \mathrm{N}, 73^{\circ} 53^{\prime} 36,12$ ” O), el cual también alcanza los 3.550 m.s.n.m. Habita laderas rocosas de acantilados altos expuestos a vientos fuertes, frecuentemente envueltos en nubes densas.

Comparación: en la Tabla 2 se presenta una comparación entre la especie Puya loca y las demás especies presentes en los alrededores de Bogotá según J. Betancur (2000). Otras especies de Puya con flores de color semejante al púrpura intenso de Puya loca son: Puya coerulea Lindl. (Chile), Puya harmsii (A.Cast.) A.Cast. (Argentina), Puya macrura Mez (Perú) y Puya meziana Wittm. (Bolivia).

Etimología: el nombre de la especie rinde honor a la 'puya loca', canción popular del Caribe colombiano compuesta por Diofante Jiménez Robles, del conjunto folclórico 'Cumbia Soledeña', que se interpreta al compás de la 'puya', un subgénero musical de la cumbia caracterizado por su ritmo rápido y jocoso. El epíteto específico proviene del adjetivo femenino 'loca', utilizado algunas veces como sinónimo de excéntrica, y que se refiriere al color inusual de sus flores.

Fenología: florece en la época de lluvias, entre los meses de noviembre y diciembre. Entre las otras especies reportadas para la región (Betancur, 2000), solo Puya nítida y Puya santosii florecen en sincronía con P. loca.

\section{Valoración de la conservación según la Unión Internacional para la Conservación de la Naturaleza}

Hasta ahora esta especie se ha encontrado en dos localidades solo en los puntos más elevados de páramos aislados aledaños. A pesar de que una de estas localidades está protegida por la reserva privada Tierraleja, y que la otra forma parte de la franja de amortiguación del Parque Nacional Natural Chingaza, la especie se puede considerar en peligro crítico (CR) dada su pequeña área de distribución naturalmente fragmentada (Unión Internacional para la Conservación de la Naturaleza - UICN, 2001; Betancur \& García, 2006).

\section{Comentarios}

Puya loca es sin duda una especie icónica del género Puya dadas sus flores de color púrpura intenso. Es extraño que encontrándose muy cerca de la capital de Colombia, donde se han concentrado las exploraciones botánicas desde los tiempos de la Colonia (Betancur, 2000; Pedraza, et al., 2001, Madriñán, 2010), esta especie haya pasado desapercibida. Probablemente esto se deba a su muy restringida área de distribución y a que su floración se limita a un corto periodo durante la época de lluvias en los meses de noviembre y diciembre.

Las observaciones en campo nos permitieron establecer al menos dos de sus potenciales polinizadores, los colibríes Colibri coruscans (Sparkling violetear) y Pterophanes cyanopterus (Great sapphirewing) (según determinación de Juan P. López). Dado su tamaño relativamente grande y su aleteo lento, este último se ve perchando perezosamente, a la manera de los trepatroncos, sobre las flores marchitas inferiores (Figura 6). Ambas especies de colibríes presentan una distribución andina, con una franja altitudinal que abarca desde los 2.500 hasta los $3.500 \mathrm{~m}$ aproximadamente; son nectarívoras-insectívoras y altamente eficientes en el proceso de polinización, especialmente en la vegetación de bosques altoandinos y páramo (Juan P. López, comunicación personal).

El hábitat típico de la especie, acantilados rocosos altos, y las espinas frágiles de las hojas, sugieren una adaptación de escape a la herbivoría del oso de anteojos (T. ornatus) comúnmente observada en otras especies simpátricas de Puya.

La presencia de una población en el páramo aledaño de Buenos Aires (ver Materiales y métodos y Figura 2), con caracteres intermedios entre $P$. loca (con pétalos de color púrpura intenso) y $P$. nítida (con pétalos de color verde pálido), en particular en cuanto al color lila de sus pétalos, sugiere la existencia de un proceso de hibridación natural entre estas dos especies (Figura 7). Después de llevar a cabo los ensayos de germinación de estas dos especies y del híbrido putativo, se obtuvo evidencia adicional de este híbrido natural; los resultados incluyeron una germinación nula del híbrido putativo y un alto porcentaje de germinación de las semillas de individuos de las dos especies parentales. 


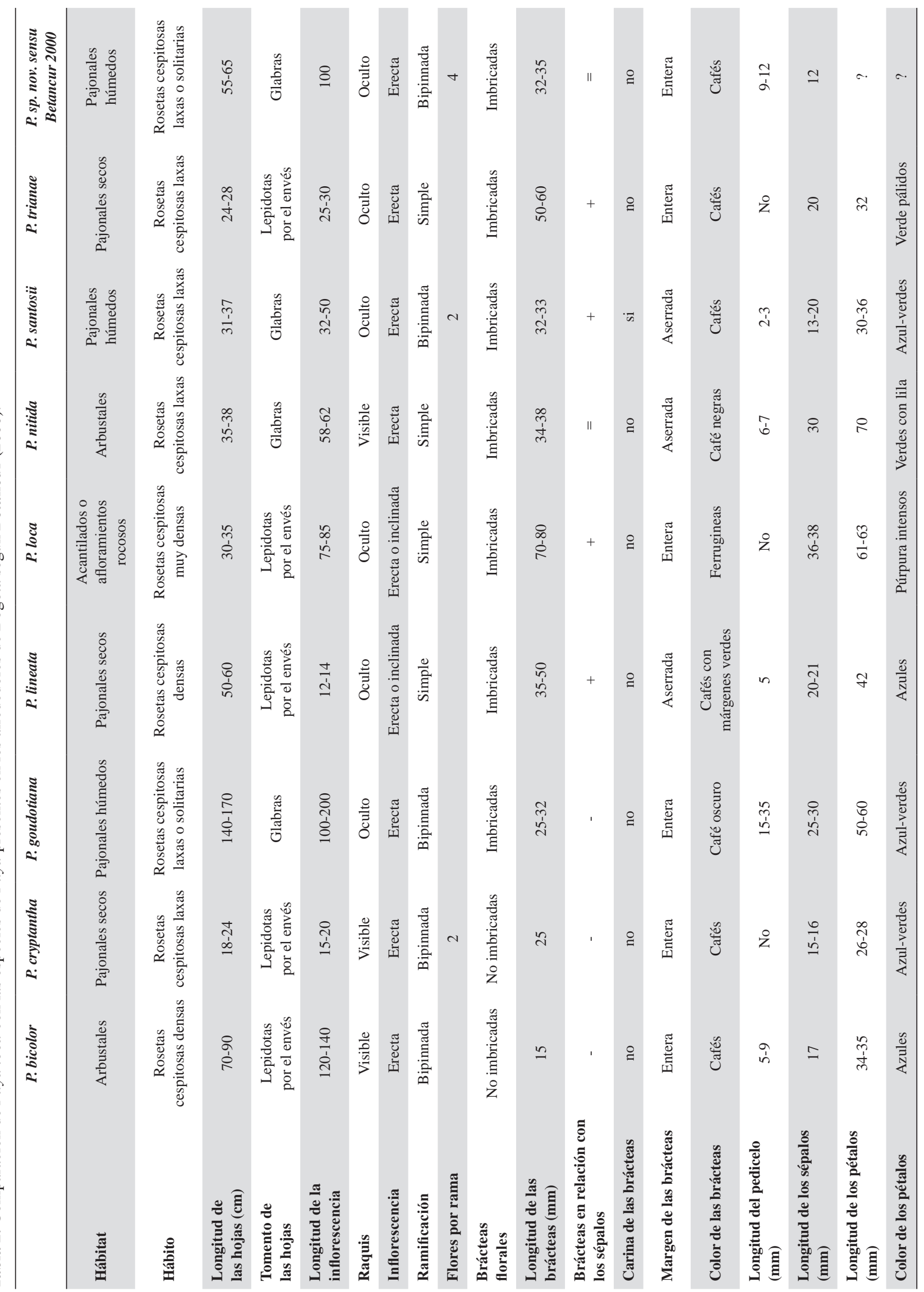



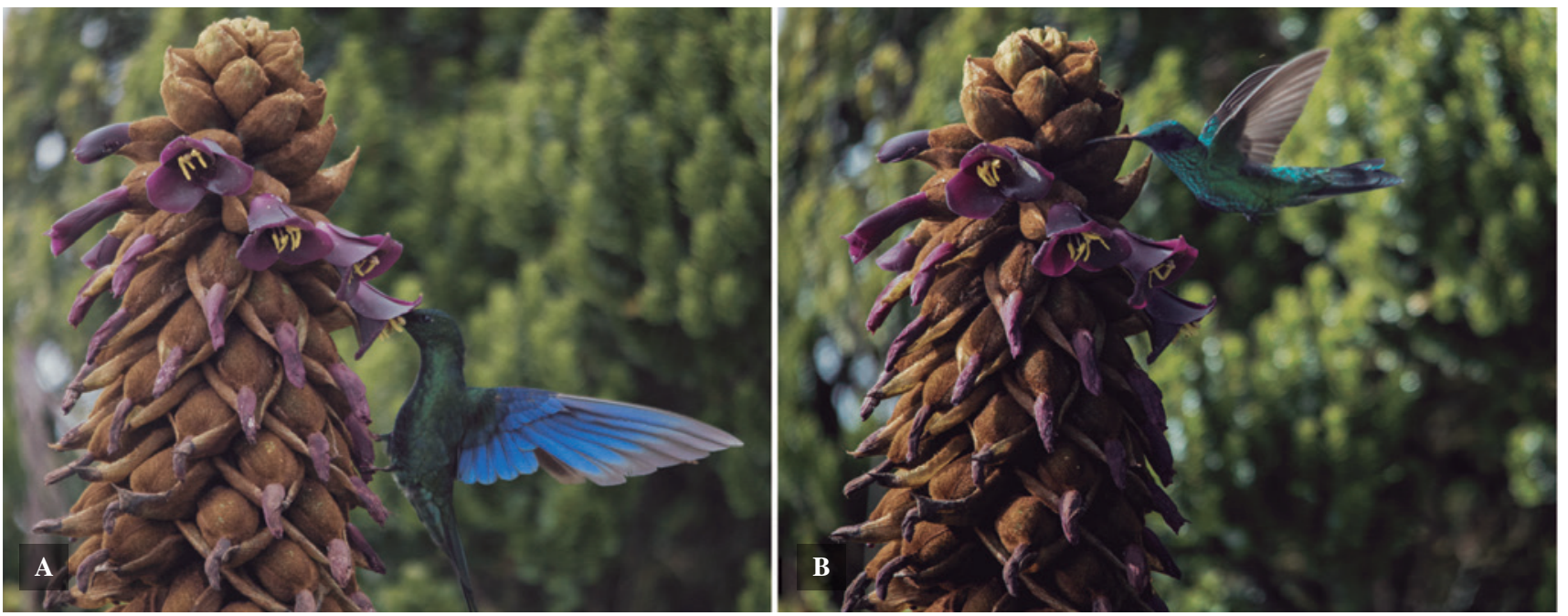

Figura 6. Polinizadores observados en el cerro Tunjaque. A. Pterophanes cyanopterus (macho). B. Colibri coruscans. Fotografías de J. P. López-Ordóñez.

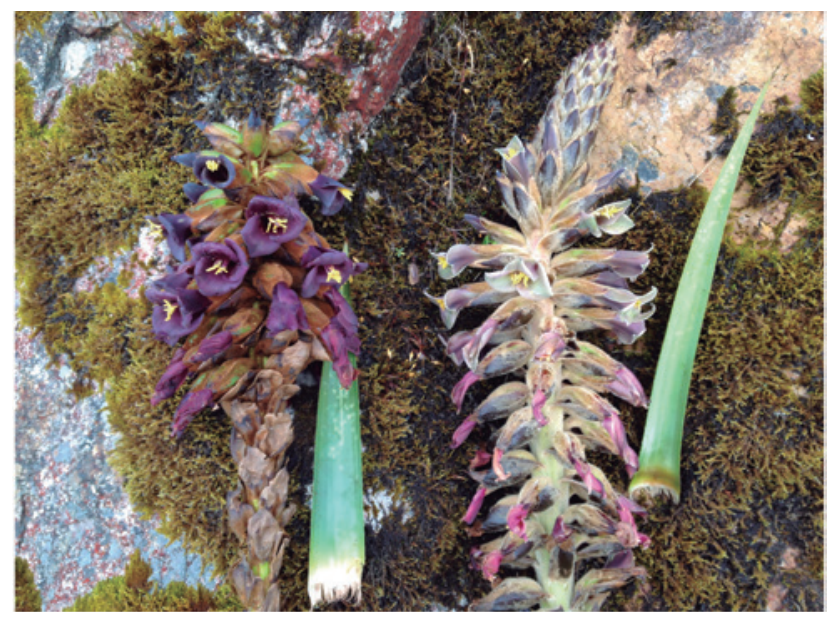

Figura 7. Montaje comparativo de especímenes presentes en la población de Cerro Verde que muestra, a la izquierda, una hoja y la inflorescencia de un espécimen introgreso de Puya loca y, a la derecha, el híbrido putativo con P. nítida. Escala: $10 \mathrm{~cm}$. Fotografía de S. Madriñán.

\section{Agradecimientos}

Mis más sinceros agradecimientos y aprecio a Antonio Piñeros, por su amable atención al permitirnos el acceso a la reserva Tierraleja, cerro Tunjaque, lo que, gracias a esas maravillosas coincidencias de la vida, en este caso auspiciadas por Emilia Restrepo, nos permitió encontrar esta nueva especie. Agradezco asimismo a los miembros del equipo del proyecto de delimitación de páramos: Sandy B. Arroyo, Ghislaine Cárdenas, Mateo Fernández, Luis F. Henao, Juan P. López, Diego Martínez, Juan S. Mendoza, Ariadna Mondragón, Karen L. Pulido y Nicolás Rodríguez, por su valiosa colaboración y amable convivencia en campo. A Kanchi N. Gandhi, del herbario de Harvard, por su incondicional apoyo en temas de nomenclatura. A Juan P. López, nuevamente, por las fotografías de los colibríes y su determinación. A Alma y Maya Gutiérrez, quienes me ayudaron con las mediciones de los caracteres morfométricos de las flores. Gerardo A. Aymard-Corredor, Ana María Bedoya, Antoine M. Cleef y Gustavo Romero revisaron las versiones iniciales del manuscrito; a ellos, mil gracias por sus correcciones y comentarios.

Este trabajo fue financiado parcialmente por el Convenio Interadministrativo No. 13-014 - FA No. 005 de 2013) del Fondo de Adaptación y el Instituto de Investigación de Recursos Biológicos "Alexander von Humboldt" en el marco del convenio No. 14-13-014-171CE con la Universidad de los Andes.

\section{Conflicto de intereses}

El autor declara no tener conflicto de intereses.

\section{Bibliografía}

Betancur, J. (2000). Guía de las Bromelias de Bogotá y sus alrededores. Alcaldía Mayor de Bogotá D.C. - DAMA, Bogotá.

Betancur, J. (2015). Puya. Catálogo de plantas y líquenes de Colombia. En: Bernal, R., Gradstein, S. R. \& Celis, M. (editores), Instituto de Ciencias Naturales, Universidad Nacional de Colombia, Bogotá. http:// catalogoplantascolombia.unal.edu.co.

Betancur, J. \& Callejas, R. (1997). Sinopsis del género Puya (Bromeliaceae) en el Departamento de Antioquia. Caldasia. 19 (1-2): 71-82.

Betancur, J. \& García, N. (2006). Las Bromelias. En: García, N. \& Galeano, G. (editores), Libro Rojo de plantas de Colombia. Instituto Alexander von Humboldt, Instituto de Ciencias Naturales de la Universidad Nacional de Colombia, Ministerio de Medio Ambiente, Vivienda y Desarrollo Territorial, Bogotá. 
Butcher, D. \& Gouda, E.J. (2015). The New Bromeliad Taxon List. University Botanic Gardens, Utrecht. http://botu07. bio.uu.nl/bcg/taxonList.php.

DeMay, S.M., Roon, D.A., Rachlow, J.L., Cisneros, R. (2014). Selective foraging on bromeliads by Andean bears in the Ecuadorian páramo. Ursus 25 (2): 139-147.

Givnish, T., Millam, K., Berry, P., Sytsma, K. (2007). Phylogeny, adaptive radiation, and historical biogeography of Bromeliaceae inferred from $n d h F$ sequence data. Aliso 23: 3-26.

Gouda, E.J. (1998). Bromeliaceae - Character list. http://botu07. bio.uu.nl/brom/chars.txt

Gouda, E.J., Butcher, D., Gouda, C.S. (2015). Encyclopaedia of Bromeliads, Version 3. University Botanic Gardens, Utrecht. http://encyclopedia.florapix.nl

Hornung-Leoni, C.T., González-Gómez, P.L., Troncoso, A.J. (2013). Morphology, nectar characteristics and avian pollinators in five Andean Puya species (Bromeliaceae). Acta Oecologica 51: 54-61.

Jabaily, R.S. \& Sytsma, K.J. (2010). Phylogenetics of Puya (Bromeliaceae): Placement, major lineages, and evolution of Chilean species. American Journal of Botany 97: 337-356.

Jabaily, R.S. \& Sytsma, K.J. (2012). Historical biogeography and life-history evolution of Andean Puya (Bromeliaceae) Botanical Journal of the Linnean Society. 171: 201-224.

Mabberley, D.J. (1987). The plant-book: A portable dictionary of the vascular plants. Cambridge University Press, Cambridge.

Madriñán, S. (2010). Flora ilustrada del Páramo de Chingaza: guía de campo de plantas comunes. Segunda edición. Ediciones Uniandes, Bogotá. http://chingaza.uniandes.edu. co/guia2010.htm
Madriñán, S., Cortés, A.J., Richardson, J.E. (2013). Páramo is the world's fastest evolving and coolest biodiversity hotspot. Frontiers in Genetics 4: 1-7.

Molina, G.I. (1782). Saggio sulla Storia Naturale del Chili. Stamperia di S. Tommaso d' Aquino, Bologna. Pp. 160161, 351.

Pedraza, P., Franco-Rosselli, P., Betancur, J. (2001). Lista comentada de las angiospermas de Chisacá (Sumapaz, Colombia). Caldasia 23: 247-259.

Rivadeneira-Canedo, C. (2008). Estudio del oso andino (Tremarctos ornatus) como dispersor legítimo de semillas y elementos de su dieta en la región de ApolobambaBolivia. Ecología en Bolivia 43: 29-39.

Scharf, U. \& Gouda, E.J. (2008). Bringing Bromeliaceae back to homeland botany. Journal of the Bromeliad Society. 58: $123-129$.

Schulte, K., Silvestro, D., Kiehlmann, E., Vesely, S., Novoa, P., Zizka, G. (2010). Detection of recent hybridization between sympatric Chilean Puya species (Bromeliaceae) using AFLP markers and reconstruction of complex relationships. Molecular Phylogenetics and Evolution 57 (3): 1105-1119.

Smith L.B. \& Downs R.J. (1974). Flora Neotropica Monograph 14, part 1: Pitcairnioideae (Bromeliaceae). Hafner Press, New York, New York.

Unión Internacional para la Conservación de la Naturaleza. (2001). Categorías y Criterios de la Lista Roja de la UICN: Versión 3.1. Comisión de Supervivencia de Especies de la UICN. UICN, Gland, Suiza y Cambridge. http://www. iucnredlist.org/static/categories_criteria_3_1. 